\title{
Editorial: Rewriting Nuclear Physics textbooks: 30 years with radioactive ion beam physics
}

Published online: 23 January 2017 - C Società Italiana di Fisica / Springer-Verlag 2017

This collection of articles contains the lectures presented at the Summer School Re-writing Nuclear Physics textbooks: 30 years of radioactive ion beam physics, which was held at the INFN, Sezione di Pisa and Department of Physics of the University of Pisa during the week 20-24 July 2015. The scope of the activity was twofold. First we celebrated thirty years since the publication of the pioneer papers (I. Tanihata et al., Phys. Rev. Lett. 55, 2676 (1985) and P.G. Hansen, B. Jonson, Europhys. Lett. 4, 409 (1987)) in which radioactive ion beams (RIB's) were used to study properties of atomic nuclei. Since then Low Energy Nuclear Physics (LENP) research fed by experiments at various facilities all over the world has experienced a great revival supported by widespread theoretical efforts which have changed deeply our understanding of nuclei and their interactions. The initiators of the field, Björn Jonson and Isao Tanihata were special guests and lecturers at the school.

The second scope of the event was to attract and educate the best possible students introducing them to the wonders of Physics with RIB's. We tried to convey to such students a view of the rich variety of on-going activities in the field, both experimental and theoretical, so that the progress made in the last thirty years can be developed further in the future with the help of the new generation of bright scientists.

The activities were directed towards students who were in the process of deciding what graduate studies to specialize on. Those students were selected and granted scholarships on the basis of their profit and interest in Nuclear Physics. The activity consisted of twelve lectures. Each lecture covered a topic contained in a standard Nuclear Physics textbook extended to show how our understanding has deeply changed due to the experience accumulated with RIB physics since 1985. In order to give the activity a fully international character, and because we aimed at favoring the participation of really young students, the first half day we proposed some introductory lectures given in separated sessions in different languages: Italian, Chinese, English, French, Spanish. Each day ended with tutorial classes in different languages. Lecturers were invited to participate in order to answer the questions by the students. There were also presentations about the possibilities to study abroad given by representatives of different countries and/or laboratories. The last day we visited the INFN Legnaro National Laboratory where SPES, the Italian RIB's facility is under construction and the students were given an introduction to the project.

The format of the school was unique, new and original. There were 150 participants of which about 100 were undergraduate students. Such a large participation represented a great success for our community. In the following we describe briefly the content of each lecture. We hope they will be instructive for many students and colleagues all over the word, now and for several years to come.

In his paper Nuclear Physics with RIB's: How it all started, Isao Tanihata explains how RIB's were created 30 years ago at the Bevalac facility in Berkeley and how neutron halos were discovered. The experiments showed that the sizes of ${ }^{11} \mathrm{Li}$ and ${ }^{6} \mathrm{He}$ were larger than than those of neighbouring, more strongly bound nuclei. These experiments gave rise to a whole new field of research. Now a huge variety of RIB's can be produced which are employed to perform very interesting research on nuclei far from stability.

In his paper, Making radioactive ion beams - Detecting reaction products, Riccardo Raabe presented in a rather extensive fashion the different production methods and the key features of some of the new experimental facilities. It is clear from his paper that several efforts are being made worldwide to push much further this frontier research. For this purpose new cutting edge techniques are being developed for the beam production and for the detection systems. 
Global properties of atomic nuclei were presented by M. Kowalska. Her lecture notes contain a very nice overview of the experimental techniques in this domain and their evolution over the past 30 years. The paper summarizes the general features of nuclear masses and radii and describes the methods used to measure these properties for stable isotopes. In recent years many of these techniques have been refined and new ones have been developed allowing the limits of sensitivity and precision to be pushed forward and the global properties of unstable nuclei to be investigated. Indeed, precise knowledge of the masses and radii of atomic nuclei is shedding light on the modifications of the strong force in the nuclear medium. State-of-the-art approaches, like laser spectroscopy and measurements of interaction cross sections, respectively, for obtaining information on charge and matter radii, and Penning traps, storage rings, and multi-reflection time of flight setups for mass measurements are nicely described.

The physics of Strong, weak and electromagnetic forces at work in atomic nuclei, decay properties were presented by G. Benzoni. This paper consists of a very useful survey of the basic properties of the decay of unstable nuclei, with a focus on $\alpha$ - and $\beta$-decay, followed by $\gamma$-decay. Special emphasis is given to super-allowed $\beta$-decays and to their connection to the Cabbibo-Kobayashi-Maskawa matrix, describing the mixing between quark weak states and quark mass states in the three fermion families. Double- $\beta$-decay is also presented with a focus on neutrinoless double- $\beta$-decay, which, once it is discovered, will verify the assumption that the neutrino is a Majorana particle, i.e. that antineutrino and neutrino are actually the same particle. Exotic decay modes and examples of recently measured decays in exotic nuclei and a list for a further reading close this nice paper.

The lecture Structure models: From shell model to ab initio methods by Sonia Bacca presents some recent theoretical results on the structure of light-to-medium mass exotic nuclei starting from first principles. Emphasis is given to comparison with experiment and what can be learned about three-body forces. The lecturer discusses the most advanced techniques for studying continuum properties.

R.J. Charity's paper on Resonance phenomena: From compound nucleus decay to proton radioactivity gives a very nice account of resonance phenomena in nuclei presented in a way which is appropriate for young students and researchers who are approaching the field for the first time. The basic theory is developed first for time-dependent decaying states in a model potential, then as solution of a scattering problem. There are many examples of recent experimental work on nuclei far from and beyond the nuclear stability line.

The subject of Nuclear structure from direct reactions with rare isotopes: observables, methods and highlights was discussed by Alexandre Obertelli. The notes discuss theoretical and experimental methods to use transfer and breakup reactions as spectroscopic tools to study structure properties of single particle states. It compares in particular the results of the two methods and their consistency. Furthermore it gives insight on the most advanced detectors and experimental techniques.

Reaction theory and its relation to LENP was introduced by Stefan Typel. This paper discusses basic scattering theory and its main application to nuclear reaction theory. There are a number of examples on the analysis of experiments aiming at extracting nuclear astrophysics reaction rates with indirect and surrogate methods and transfer at very low energy.

Spins in exotic nuclei: RI beam experiments with polarized targets by T. Uesaka. The lecture was devoted to show how the nuclear spin-degrees of freedom contribute to the nuclear properties and how they evolve far from the $\beta$-stability valley. Initially it is discussed how the spin-dependent interactions originate; in particular, how the first pioneering ideas promoted to explain the strong nuclear spin-orbit coupling developed towards the modern interpretation in terms of two-nucleon, three-nucleon and tensor interactions. Then, a special emphasis is given to the valuable opportunities opened by the use of RIB's in experiments with polarized targets of light nuclei, such as H and He. A glance to the recent developments on the production and handling of gaseous polarized targets concludes the lecture.

Single particle versus collectivity, shapes of exotic nuclei by Andrea Jungclaus. In this article some selected topics of nuclear structure research have been discussed as illustration of the progress reached in this field during the last thirty years. These examples evidence the improvement of our understanding of the atomic nucleus obtained on the basis of countless experiments, performed to study both exotic nuclei (nuclei far off the valley of stability) as well as nuclei under exotic conditions (high excitation energy/temperature or large angular momentum/rotational frequency), using stable and radioactive ion beams. The paper also gives many examples, including light dripline nuclei, two-proton decay of ${ }^{54} \mathrm{Fe}$, isospin symmetry in heavier nuclei, and prompt proton emission from a deformed state of ${ }^{58} \mathrm{Cu}$.

Radioactive ion beams in nuclear astrophysics by Lucio Gialanella. This lecture shows how unstable nuclei play a crucial role in the Universe. In this lecture, after a short introduction to the field of Nuclear Astrophysics, few selected cases in stellar evolution and nucleosynthesis are discussed to illustrate the importance and peculiarities of processes involving unstable species. Finally, some experimental techniques useful for measurements using radioactive ion beams and the perspectives in this field are presented.

The paper on What's next in nuclear physics with RIB's was delivered by B. Jonson. His lecture notes give a nice account of the discovery of the nuclear halo and other accomplishments in the field during the 30 years that have passed since Isao Tanihata's famous experiments at Berkeley. New and upgraded facilities and experimental equipment are presented and the author remarks that: "There has never before, in the history of nuclear science, been such a rapid development of new and upgraded research facilities as we witness today." Next-generation experiments with 
new experimental probes are discussed including, just knocking at the door, the investigation of the internal nuclear structure of short-lived radioactive nuclei by means of electron scattering or by annihilation when radioactive species collide with antiprotonic atoms. Jonson ends his paper by quoting a poem by Esaias Tegner (1782-1846), regarded as the father of modern poetry in Sweden, who wrote:

This is Man's wonderful ability

to be able to grasp the inner essence of phenomena, not what they appear to be, but what they mean, and the reality that we see with our eyes

a symbol only of something higher.

Let this poem be the symbol and inspiration of this series of lectures.

Nicolas Alamanos, Carlos Bertulani, Angela Bonaccorso, Angela Bracco, David M. Brink, and Giovanni Casini 